\title{
HOW IS CLASSMATE AND PE TEACHER SUPPORT ASSOCIATED WITH THE LEVEL OF PHYSICAL ACTIVITY IN YOUNG ADOLESCENTS FROM KOSOVO? THE ROLE OF GENDER AND AGE
}

\author{
Michal Bronikowski', Ida Laudańska-Krzemińska', Małgorzata Bronikowska', Besnik Morina ${ }^{2}$ \\ ${ }^{1}$ Department of Didactics of Physical Activity, University School of Physical Education in Poznan, Poznan, Poland \\ ${ }^{2}$ Department of Didactics of Physical Education, University of Pristina, Pristina, Kosovo
}

\begin{abstract}
SUMMARY
Aim: This study aimed to investigate how were the peer and physical education (PE) teacher variables associated with moderate-to-vigorous physical activity (MVPA) among Kosovar teenagers (13-15 years of age), and the role of age and gender in these associations.

Methods: Cross-sectional data was gathered through a study conducted in seven major municipalities of Kosovo. 632 girls (aged 14.3 \pm 0.8 ) and 664 boys (aged 14.2 \pm 0.8 ) were examined using the adjusted Classmate and Teacher Support Scale and the Physical Activity Screening measure. A three-way (support ${ }^{*}$ age ${ }^{*}$ gender) ANOVA was used to compare the individuals' MVPA level with the different levels of support they received, with different age in groups of girls and boys.

Results: Boys reported higher levels of MVPA than girls in all age categories. A higher level of MVPA was reported by boys receiving high or medium support from classmates. In case of PE teacher support, high, medium and even low support correlated with high MVPA in all age categories. In girls only 13 year olds reported receiving high levels of both classmate and PE teachers support which correlated with a higher level of MVPA.

Conclusions: It appears that engagement in MVPA among young Kosovar adolescents is relatively high in boys, regardless of the level of classmate or PE teacher support, whereas in girls the level of MVPA decreased over their time at school. Support from both classmates and PE teachers turned out to be the most significant factor in 13 years old girls, before they moved from primary to secondary school. This issue requires more detailed insight, considering traditional ethnical and religious barriers to engagement of young female adolescents in physical activity.
\end{abstract}

Key words: physical activity, peer support, teacher support, adolescents

Address for correspondence: M. Bronikowski, Department of Didactics of Physical Activity, University School of Physical Education, Krolowej Jadwigi 27/38, 61871 Poznan, Poland. E-mail: bronikowski.michal@wp.pl

\section{INTRODUCTION}

It has become evident that children and adolescents throughout Europe and indeed around the world do not undertake physical activity (PA) at recommended levels (1), and that the level of involvement tends to decline with age (2). Understanding factors affecting young people's participation in PA has become an issue of great importance worldwide.

Systematic reviews have identified robust correlates associated with PA. Gender, socioeconomic status, psychological characteristics, friends, family, and physical environment are related to different activity levels (3-5). More studies are required to understand how various factors determine PA behavioural patterns, especially in children and teenagers with poor socioeconomic status and/or low social support. In a study by Petronyte and Zaborskis (6) on the factors influencing leisure time physical activity among adolescents in Europe, regional difference was one of the factors that required further research. There might also be other circumstances, such as gender or culture, specifically in regions heavily affected by social tensions based on religious and ethnic conflicts like Kosovo.

Social support from parents, friends and physical education (PE) teachers were reported as significant predictors of self- reported engagement in PA. Specifically, support from friends was found to be the most important social environmental factor in young adolescents (7). This makes sense from a developmental perspective as peer support in adolescence is generally a dominant social factor and plays a critical role in the development of PA motivation and engagement. It is worth investigating the level of such support in the context of Kosovar adolescents.

Providing the optimal level of PA and shaping health attitudes and behaviours in adolescents is a key role of school PE (8-10) and PE has also been shown to be an important mediator of PA outside school (11). Specifically, well documented girls disengagement from school PE $(12,13)$ is a cause for concern.

A low level of PA engagement can also be related to the masculine and feminine gender roles, especially within the context of religious, ethnic and cultural tradition factors. Ansari et al. (14) reported in their study on female students from Libya (a country with a strong Islamic tradition) that they are particularly at risk of low level of PA, suggesting that attention should be paid to national level policies that promote active living, along with addressing cultural, geographical and religious barriers to engagement of young (female) adolescents in PA.

With reference to the geographical focus of the paper, in a study on the post-war needs of children in Kosovo (15) it 
was found that only $12 \%$ of girls compared to $39 \%$ of boys declared participation in daily PA, whereas $20 \%$ of boys and $42 \%$ of girls declared rare or no participation in PA. This issue therefore opens some new, potentially fruitful alleys for more in-depth research.

The study required a theoretical framework that would allow a suitably robust assessment of the findings. The trans-contextual model has therefore been used, which hypothesizes that perceived autonomy support in PE would be transferred into autonomous motivation in non-educational, after school settings. This model is grounded in self-determination theory (16), which suggests that social factors support self-determination motivation and associated positive outcomes when psychological needs for competence, autonomy, and relatedness are met. Differences in the traditional roles of men and women in social life of such a unique region as the Balkans, combined with the diachronic context (post-war, post-conflict), required the use of cross-cultural research approaches and a social ecological perspective (17).

In the 1990s, during the years of conflict, Kosovar children were denied the right to study in schools and relied on the ad hoc 'underground' education (18). Therefore, reliable research is required into the PE system of Kosovo in order to provide interventions directed explicitly at improving children's PE experiences. Social support has been recognized for its influencing role in motivating youth (19). Furthermore, findings concerning potential mediators of teenage MVPA, specifically in Kosovo, a region barely investigated in this matter, will inform future interventions on mechanisms of health-related behaviour change.

Therefore, the objective of this study was to improve understanding of the factors associated with undertaking and maintenance of PA during early teenage years. Following findings from other research, we designed a study to investigate the associations of classmate and teacher support during PE lessons on MVPA of 13-15 years old girls and boys from Kosovo. We also took into account the role of gender and age.

\section{MATERIALS AND METHODS}

\section{Participants}

The cross-sectional sample for the study included data from 664 boys aged $13-15$ years $(M=14.2 \pm 0.8)$ and 632 girls aged $13-15$ years $(M=14.3 \pm 0.8)$, examined in 2014. The participants were recruited from randomly selected secondary schools in the seven major municipalities of Kosovo. Each of the municipalities had a population of more than 70,000 people: Pristina (102 boys, 102 girls), Mitrovica ( 92 boys, 110 girls), Peja (95 boys, 90 girls), Gjakova (95 boys, 90 girls), Prizren ( 95 boys, 88 girls), Gjilan (94 boys, 100 girls), and Ferizaj (91 boys, 102 girls). The sample of students was selected through proportional stratified sampling. The sample unit was the school. All participants (and their parents) received a letter describing the purpose and procedure of the study. Students were also informed about the anonymous and voluntary nature of their participation, that the study records would be kept confidential, and that their individual contributions would be unidentifiable in the final report. Questionnaires were completed in whole-class groups during one regular school lesson in quiet classroom conditions and took approximately 20 minutes to complete. The non-response rate (due to either parental disapproval or faulty questionnaire completion) was $3 \%$.

\section{Classmate and Teacher Support Measure}

In case of external support, two scales containing five questions each were designed to assess classmate and teacher support during PE lessons. These scales were based on the Classmate and Teacher Support Scale, and the test-retest correlations were 0.69 (20). Based on results from 11-16 years old girls and boys from seven different countries Torsheim et al. (21) indicated that the Classmate and Teacher Support Scale is well suited for use in large social surveys and can be used in spite of potential language and cultural differences.

In this study, it was decided to adjust the original Classmate and Teacher Support Scale so that it was fully applicable to the PE environment. The internal consistency of the scales was established using Cronbach's Alpha test. For the Teacher support scale $\alpha=0.91$, and for the Classmate support $\alpha=0.87$ (22). Finally, the statements on the classmate support scale were as follows: 1 - other students accept me as I am, 2 - most of the students in my class are kind and helpful, 3 - the students in my class enjoy being together, 4 - I get positive feedback from my peers when I play, and 5 - I am often picked to play on various teams. Statements 1,2, and 3 came from the original questionnaire (20) and the remaining statements were designed by the researchers. The items on the teacher support scale were: 1 - our teacher treats us fairly, 2 - when I need extra help, I can get it, 3 - I get positive feedback from my teacher when I play, 4 - our teacher makes sure we all treat one another fairly, and 5 - the teacher lets us express our opinions. Statements 1 and 2 came from the original questionnaire (20) and the other three were designed by the researchers. A panel of international experts (from Poland, Kosovo, England, and Austria), who were cooperating on the EU project EuropeAid/130886/C/SER/KOS, evaluated both instruments and translated the English version of the instruments into Albanian.

The examined individuals were required to assess, on a 5-point Likert scale, whether they agree (strongly agree) or disagree (strongly disagree) with each statement. The total possible score was 25 points on each of the scales. Categorization of support from teachers and classmates was made with the use of individual scores normalized to a sten scale (23), where individuals with 1-4 sten scores were classified as exhibiting a low level of support, those with scores of 5-6 as having a medium level of support, and individuals with 7-10 sten scores as receiving high support.

\section{Physical Activity Measure}

The level of PA was determined using the Physical Activity Screening Measure (24). This measure corresponds to the average number of days per week with at least 60 minutes spent partaking in various forms of PA during which, in the participants' opinion, their heart rates increased and they experienced a feeling of shortness of breath (higher breathing frequency). Students were asked to answer two questions: P1 - Over the past 7 days, on how many days were you physically active for a total of at least 60 minutes per day?; P2 - Over a typical week, how many days are you physically active for a total of at least 60 minutes per day? The MVPA index was calculated based on the following formula: 
$\mathrm{MVPA}=(\mathrm{P} 1+\mathrm{P} 2) / 2$ where: $\mathrm{MVPA}=\mathrm{PA}$ index; $\mathrm{P} 1=$ number of physically active days during the past 7 days; $\mathrm{P} 2=$ number of physically active days during typical week.

\section{Statistical Methods}

It was examined whether, and to what extent, the level of support from PE teachers, support from classmates and age were in any way associated with the level of MVPA among girls and boys. To compare individuals' MVPA levels with the different levels of support they received (low, medium, high) with different age in groups of girls and boys, a three-way (support* age* gender) ANOVA was used. To conduct detailed multiple comparisons Tukey's HSD post-hoc test was employed. To determine the effect level for particular effects the eta-squared value was calculated. Differences between genders and age categories in classmate support or teacher support were analyzed using chi-square analysis. Cramér's V index was used as a measure of association to quantify the strengths of the relationships (25). Significance was denoted by $\mathrm{p}<0.05$. Statistical analysis was carried out using Statistica 10.0 software.

\section{RESULTS}

The descriptive statistics of the peer support in PE, teacher support in PE, MVPA, gender and age are given in Table 1 and 2. Teenage boys from Kosovo have higher MVPA than girls $(p<0.0001)$. The highest MVPA was observed in combined group of 14 years old boys and girls $(\mathrm{p}=0.0002)$. Higher classmate and teacher support on PE are related to higher MVPA $(\mathrm{p}<0.0001)$ (Table 1).

According to classmate support in PE (Table 2), teenage girls from Kosovo reported high support less frequently than boys $(p<0.001)$. A similar situation was noticed with PE teacher support $(\mathrm{p}=0.0024)$. There was a noticeable differentiation between age groups in classmate support $(\mathrm{p}=0.0037)$ and teacher support $(p=0.0022)$. The most frequent high classmate support in PE was indicated by 15 years old Kosovar teenagers. The low classmate support in PE was most frequently reported by 13 year olds. Conversely, a diametrically opposite situation was observed in teacher support in PE. High teacher support in PE was indicated
Table 1. Mean and standard deviation of MVPA for analyzed variables

\begin{tabular}{|c|c|c|c|}
\hline Variables & $\begin{array}{l}\text { MVPA } \\
M \pm S D\end{array}$ & $p$ value & Eta-squared \\
\hline \multicolumn{4}{|l|}{ Gender } \\
\hline Boys & $3.68 \pm 1.49$ & \multirow{2}{*}{$<0.0001$} & \multirow{2}{*}{0.10} \\
\hline Girls & $2.77 \pm 1.23$ & & \\
\hline \multicolumn{4}{|c|}{ Age groups } \\
\hline 13 & $3.26 \pm 1.44$ & \multirow{3}{*}{0.0002} & \multirow{3}{*}{0.01} \\
\hline 14 & $3.47 \pm 1.46$ & & \\
\hline 15 & $3.07 \pm 1.41$ & & \\
\hline \multicolumn{4}{|c|}{ Classmate support } \\
\hline Low & $2.83 \pm 1.26$ & \multirow{3}{*}{$<0.0001$} & \multirow{3}{*}{0.04} \\
\hline Medium & $3.27 \pm 1.44$ & & \\
\hline High & $3.55 \pm 1.5$ & & \\
\hline \multicolumn{4}{|c|}{ Teacher support } \\
\hline Low & $3.10 \pm 1.53$ & \multirow{3}{*}{$<0.0001$} & \multirow{3}{*}{0.02} \\
\hline Medium & $3.16 \pm 1.36$ & & \\
\hline High & $3.54 \pm 1.48$ & & \\
\hline
\end{tabular}

most often by 13 years old Kosovar teenagers and low classmate support in PE was indicated most often by 15 years old teenagers (Table 2).

In the present study the interaction effect of three factors: classmate support in PE (low, medium, high), gender (boys, girls) and age $(13,14,15$ years old) on MVPA of Kosovar adolescents was also analyzed (Table 3). Using MVPA as the dependent measure, a three-way ANOVA (classmate support* gender* age) revealed a significant key effect of the level of classmate support in $\mathrm{PE}(\mathrm{F}(2$, $1278)=86.16$; $\mathrm{p}<0.0001)$ - with those receiving lower support having lower MVPA, gender $(\mathrm{F}(1,1278)=153.87$; $\mathrm{p}<0.0001)-$ girls have lower MVPA $(\mathrm{p}<0.0001)$, and age $(\mathrm{F}(2,1278)=3.65$; $\mathrm{p}=0.0262)-14$ year olds have higher MVPA than 15 years old teenagers $(p<0.0001)$. The results also indicated a significant two-way interaction effect for classmate support * age $(\mathrm{F}(4,1278)$ $=3.19 ; \mathrm{p}=0.0128$ ). 13 years old Kosovars with low classmate support also had lower MVPA than their peers with medium $(p=0.0157)$ and high $(p<0.0001)$ support. A similar effect, albeit

Table 2. Descriptive statistics for classmate and teacher support in PE

\begin{tabular}{|c|c|c|c|c|c|c|c|c|c|}
\hline \multirow[b]{2}{*}{ Variables } & \multirow{2}{*}{$\begin{array}{c}\text { All } \\
\mathrm{N}(\%)\end{array}$} & \multicolumn{3}{|c|}{ Classmate support } & \multirow[b]{2}{*}{ Cramér's V } & \multicolumn{3}{|c|}{ Teacher support } & \multirow[b]{2}{*}{ Cramér's V } \\
\hline & & $\begin{array}{l}\text { Low } \\
\mathrm{n}(\%)\end{array}$ & $\begin{array}{c}\text { Medium } \\
\mathrm{n}(\%)\end{array}$ & $\begin{array}{l}\text { High } \\
\mathrm{n}(\%)\end{array}$ & & $\begin{array}{l}\text { Low } \\
\mathrm{n}(\%)\end{array}$ & $\begin{array}{c}\text { Medium } \\
\mathrm{n}(\%)\end{array}$ & $\begin{array}{l}\text { High } \\
\mathrm{n}(\%)\end{array}$ & \\
\hline \multicolumn{10}{|l|}{ Gender } \\
\hline Girls & $632(48.8)$ & $224(35.5)$ & $248(39.2)$ & $160(25.3)$ & \multirow{2}{*}{$0.18^{* *}$} & $152(24.1)$ & $340(53.8)$ & $140(22.1)$ & \multirow{2}{*}{$0.10^{*}$} \\
\hline Boys & $664(51.2)$ & $144(21.7)$ & $262(39.5)$ & $258(38.9)$ & & $154(23.2)$ & $308(46.4)$ & $202(30.4)$ & \\
\hline \multicolumn{10}{|c|}{ Age groups } \\
\hline 13 & $318(24.5)$ & $110(34.6)$ & $126(39.6)$ & $82(25.8)$ & \multirow{3}{*}{$0.08^{*}$} & $66(20.7)$ & $156(49.1)$ & $96(30.2)$ & \multirow{3}{*}{$0.08^{*}$} \\
\hline 14 & $386(29.8)$ & $114(29.5)$ & $152(39.4)$ & $120(31.1)$ & & $72(18.7)$ & $204(52.9)$ & $110(28.5)$ & \\
\hline 15 & $592(45.7)$ & $144(24.3)$ & $232(39.2)$ & $216(36.5)$ & & $168(28.4)$ & $288(48.7)$ & $136(23.0)$ & \\
\hline Total & $1296(100)$ & $368(28.4)$ & $510(39.4)$ & $418(32.3)$ & & $306(23.6)$ & $648(50.0)$ & $342(26.4)$ & \\
\hline
\end{tabular}


with a smaller statistical significance level, was noticed among 14 and 15 years old teenagers: those with low classmate support had lower MVPA than those with high support $(\mathrm{p}<0.0001, \mathrm{p}=0.0218$, respectively). The results also indicated a significant two-way interaction effect for gender $*$ age $(F(2,1278)=3.44 ; p=0.0324)$. However, post-hoc analyses using Tukey's HSD indicated no differentiation between age groups among boys or girls.

The interaction effect (classmate support* gender*age) was significant $(F(4,1278)=3.32 ; p=0.0103$; eta-squared $=0.01)$. The Figure 1 shows these interactions. Among 13 years old girls a differentiation in MVPA according to classmate support was noticed. Post-hoc analyses using Tukey's HSD indicated that girls who received higher classmate support had significantly higher MVPA than those with medium $(p=0.0320)$, and low $(p=0.0002)$ support.

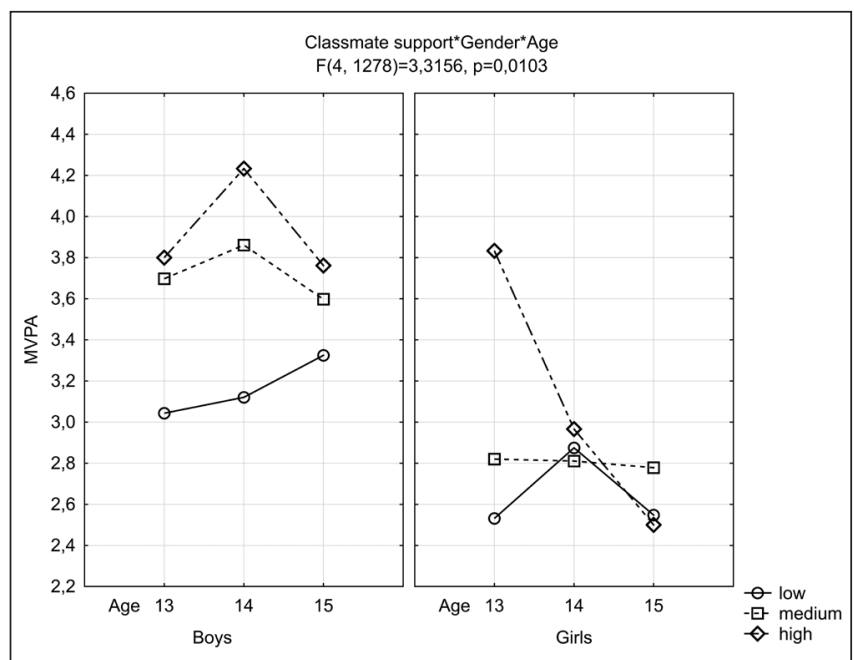

Fig 1. Three-way ANOVA (classmate support in $P E^{*}$ gender age) interaction effect.
Among 14 and 15 years old girls there were no such interactions ( $p>0.99 ; p>0.99$, respectively). Male teenage respondents also differed in a supplementary way and the most sensitive age appeared to be 14. Boys at that age, who received higher classmate support in PE had significantly higher MVPA than those with low support $(\mathrm{p}=0.0001)$. There were no such interactions among 13 $(p>0.44)$ and $15(p>0.95)$ years old boys.

The interaction effect of teacher support in PE, gender, and age on MVPA of Kosovar teenagers was also analyzed (Table 4).

Using MVPA as the dependent measure, a three-way ANOVA (teacher support* gender*age) revealed a significant effect of the level of teacher support in $\operatorname{PE}(F(2,1278)=6.24 ; p=0.0020)$ - teenagers with high level of support had significantly higher MVPA from those with medium $(\mathrm{p}=0.0001)$, and low $(\mathrm{p}<0.0001)$

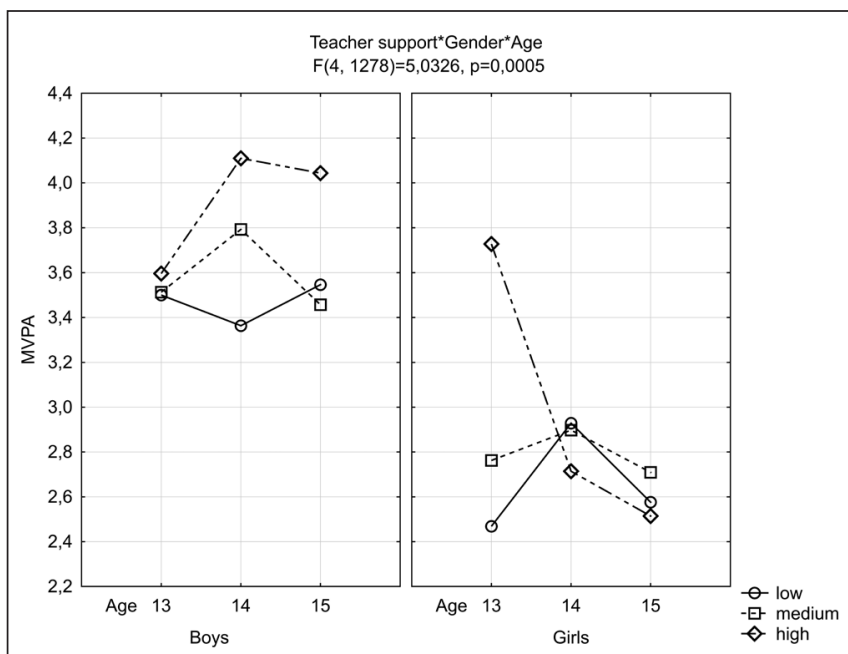

Fig. 2. Three-way ANOVA (teacher support in $P E^{*}$ gender age) interaction effect.

Table 3. Statistics of three-way ANOVA (classmate support in $P E^{*}$ gender ${ }^{*}$ age) on MVPA

\begin{tabular}{|l|c|c|c|}
\hline Source & F & p value & Eta-squared \\
\hline Classmate support & 17.251 & $<0.0001$ & 0.0262 \\
\hline Gender & 86.164 & $<0.0001$ & 0.0631 \\
\hline Age & 3.651 & 0.0262 & 0.0056 \\
\hline Classmate support ${ }^{*}$ gender & 2.336 & 0.0971 & 0.0036 \\
\hline Classmate support ${ }^{*}$ age & 3.189 & 0.0128 & 0.0098 \\
\hline Gender ${ }^{\text {age }}$ & 3.437 & 0.0324 & 0.0053 \\
\hline Classmate support ${ }^{*}$ gender ${ }^{*}$ age & 3.316 & 0.0103 & 0.0102 \\
\hline
\end{tabular}

$R^{2}=0.15 ; R^{2}(\operatorname{adj})=0.14$

Table 4. Statistics of three-way ANOVA (teacher support in $P E^{*}$ gender*age) on MVPA

\begin{tabular}{|l|c|c|c|}
\hline Source & F & p value & Eta-squared \\
\hline Teacher support & 6.237 & 0.0020 & 0.0096 \\
\hline Gender & 98.992 & $<0.0001$ & 0.0718 \\
\hline Age & 1.539 & 0.2149 & 0.0024 \\
\hline Teacher support ${ }^{*}$ gender & 0.258 & 0.7728 & 0.0004 \\
\hline Teacher support*age & 1.259 & 0.2841 & 0.0039 \\
\hline Gender ${ }^{*}$ age & 3.569 & 0.0284 & 0.0055 \\
\hline Teacher support* ${ }^{*}$ gender ${ }^{*}$ age & 5.033 & 0.0005 & 0.0155 \\
\hline
\end{tabular}

$R^{2}=0.14 ; R^{2}(\operatorname{adj})=0.12$ 
support, and gender $(\mathrm{F}(1,1278)=98.99 ; \mathrm{p}<0.0001)-$ girls have lower MVPA $(\mathrm{p}<0.0001)$. The interaction effect (teacher support * gender*age $)$ was significant $(\mathrm{F}(4,1278)=5.03 ; \mathrm{p}=0.0005$; etasquared $=0.02)$. Figure 2 indicates these interactions. Among 13 years old girls the impact of teacher support on MVPA was also noticed. Post-hoc analyses using Tukey's HSD indicated that girls who received the highest teacher support had significantly higher MVPA than those with medium ( $\mathrm{p}=0.0165)$, and low $(\mathrm{p}=$ 0.0075 ) support. Among 14 and 15 years old girls there were no such interactions ( $p>0.99 ; p>0.99$, respectively). Furthermore, there was no such differentiation among boys.

\section{DISCUSSION}

The increasing percentage of adolescents in Europe failing to meet the recommended MVPA guidelines, for example in Belgium reaching $75 \%$ (26), continues to raise concerns and indicates the need for further studies to understand more clearly the factors influencing health-related behaviours. The significant inter-personal relationships (parents, teachers, peers) play a crucial role in young people's engagement in PA.

Due to entrenched cultural traditions in Kosovo, patriarchal familial relations remain the basis of social functioning and solidarity (27). More specifically, the issue of low participation in PA significantly concerns girls who are traditionally underrepresented in sport and have no enduring habits of being physically active. There is also a limited range of competitive sports in school PE curricula. This is in line with our findings: a lower level of MVPA was noticed in girls. However, this was not the case for girls aged 13 years. There were some circumstances that enabled girls to have a higher level of MVPA, namely support from PE teachers and peers. In case of PA, Belcher et al. (28) report that the most dramatic age-related decline occurs at the onset of puberty, specifically in girls, regardless of ethnicity or weight status. However, there may be other circumstances. Taking into account the paternalistic tradition of social life in Kosovo, there might be a cumulative effect of the transition from childhood into early adolescence combined with the differentiation of social roles of males and females, occurring at the moment of changing schools from primary to secondary ones, which takes place at the age of 14 in Kosovo. Furthermore, students, particularly boys, aged 14 are provided with more opportunities for competitive sports through the intra-school system of competition, which results in high MVPA. Low MVPA in girls, specifically those receiving medium or low support, may also be due to the lack of competitive motives in girls' PE experiences, and the traditionally strong masculinity factor in boys PE in Kosovo. Weinberg et al. (29) found competitive factors (competition, social/energy, fitness/fun, teamwork) to be equally strong across cultures, but more important for males than females. In our study there was a similar effect in receiving high support from PE teachers among 13 years old boys and girls resulting in higher MVPA, whereas when they change primary school to the secondary education, a higher level of PE teacher support was reported in boys only. It is possible that traditional feminine roles are already assigned to girls by the time they advance to secondary education and they are no longer treated as children, on equal terms with the boys in sporting and PA contexts.
In terms of classmate support, the highest level of MVPA in 13 years old girls was associated with receiving high support. Interestingly, a similar pattern was observed among 14 years old boys. Generally, boys declared receiving higher support than girls from both classmates and PE teachers in all age categories, but the sources of support and its level differed along the school years. Nevertheless, for 13 year olds (both boys and girls) classmate support was reported as low, whereas PE teachers support was high. This change from reliance on parental or teacher support to an increase in the influence of peer support at this age has been reported in other studies as well (7). Parents influence a child's behaviour, and fathers specifically play a role in health-related activity. This process begins in early childhood and continues until adolescence. In contrast, peer influence is believed to increase with age as children grow and mature (30). With regards to parental support for PA, children's perceptions of parental involvement differ because boys perceive more paternal than maternal tangible support (31). Fathers tend to be more engaged in playful PA with their children compared with mothers (32). Mothers seem to traditionally play a more nurturing/emotional role. Generally speaking, young boys identify more with fathers and girls with mothers (31). Therefore, parents appear to be the most influential source of support in their childhood. To reiterate, while parents continue to influence adolescents' health behaviours, the role of peer influence is believed to increase with age (33). A significant modelling role in patterns of PA involvement among young adolescents coming from a culture of strong patriarchal profile like Kosovo might be explained in such way.

In a similar manner to the declining parental influence on children as they grow up, the influence of PE teacher support also seems to fade with school years. Though, an opposite trend among classmate support in PE was noticed, thus indicating the increasing role of peer support. Our results suggest that at this stage of education there is insufficient focus on supporting girls' PA, which is probably a traditionally and culturally accepted practice. Moreover, it is a state of affairs perceived by all, i.e. students, teachers and probably parents as well. In our study this pattern was visible among 15 year olds, who declared high support from classmates, but a low level of support from PE teachers. On the other hand, in both cases it was boys who reported higher levels of support from classmates and PE teachers, which was associated with higher levels of MVPA.

Nevertheless, a more focused approach to improving the quality of PE and school sport is needed in terms of support for both boys and girls. The findings of a longitudinal study by Bunke, Apitzsch and Bäckström (34) on 16-19 years old students indicated that social support for adolescents should be offered over a longer period of time. Furthermore, social experience had a direct reductive effect and an indirect positive effect on current and intended PA. As a result, increased focus should be on linking the initially external regulation of PA to the more internal regulation as students get older. The findings from our study prove that such regulation should also be provided as early as possible in their personal growth. Elsewhere, Jurak et al. (35) have found that when an intervention is aimed at primary school pupils and extended for a period of 4 years, the initially significant differences in anthropometric measures between intervention and control groups decrease, but the groups differ significantly in all motor tasks, with experimental groups scoring higher. 
A key strength of this study is the effort to better understand the complexity of how PE teacher and peer variables relate to teenagers MVPA, specifically in the relatively unknown 'territory' of social support in the education system of Kosovo. To our knowledge, this is the first study which attempts to explain the associations between these variables and leisure time PA among Kosovar adolescents.

There are, however, some limitations. One limitation is the use of cross-sectional data, which does not allow for determining any causal associations between variables. However, the large sample size does allow for robust estimates of associations. Stronger study designs are needed to confirm the direction of relationships between PE teachers, peers and PA variables. Longitudinal research has the ability to make causal statements, but an experimental study would be more helpful in determining whether more support would affect an increase in leisure-time PA.

\section{Acknowledgements}

This study was supported by the EU project EuropeAid/130886/C/SER/ KOS.

\section{Conflict of Interests}

None declared

\section{REFERENCES}

1. Currie C, Gabhainn SN, Godeau E, Roberts C, Smith R, Currie D, et al., editors. Inequalities in young people's health: HBSC international report from the 2005/2006 survey. Copenhagen: WHO Regional Office for Europe; 2008.

2. Bradley RH, McRitchie S, Houts RM, Nader P, O'Brien M; NICHD Early Child Care Research Network. Parenting and the decline of physical activity from age 9 to 15 . Int J Behav Nutr Phys Act. 2011 Apr 15;8:33. doi: 10.1186/1479-5868-8-33.

3. Sallis JF, Prochaska JJ, Taylor WC. A review of correlates of physical activity of children and adolescents. Med Sci Sports Exerc. 2000 May;32(5):963-75.

4. Van Der Horst K, Paw MJ, Twisk JW, Van Mechelen W. A brief review on correlates of physical activity and sedentariness in youth. Med Sci Sports Exerc. 2007 Aug;39(8):1241-50.

5. Lubans DR, Foster C, Biddle SJ. A review of mediators of behavior in interventions to promote physical activity among children and adolescents. Prev Med. 2008 Nov;47(5):463-70.

6. Petronyte $\mathrm{G}$, Zaborskis A. The influence of individual and regional factors on association between leisure time physical activity and psychological complaints among adolescents in Europe. Cent Eur J Public Health. 2009 Dec;17(4):215-9.

7. Zhang T, Solmon MA, Gao Z, Kosma M. Promoting school students' physical activity: a social ecological perspective. J Appl Sport Psychol. 2012;24(1):92-105.

8. Green K. Physical education, lifelong participation and 'the couch potato society'. Phys Educ Sport Pedagogy. 2004;9(1):73-86.

9. Johns DP, Ha AS. Home and recess physical activity of Hong Kong children. Res Q Exerc Sport. 1999 Sep;70(3):319-323.

10. Sallis JF, Prochaska JJ, Taylor WC, Hill JO, Geraci JC. Correlates of physical activity in a national sample of girls and boys in grades 4 through 12. Health Psychol. 1999 Jul;18(4):410-5.

11. Barr-Anderson DJ, Young DR, Sallis JF, Neumark-Sztainer DR, Gittelsohn J, Webber L, et al. Structured physical activity and psychosocial correlates in middle-school girls. Prev Med. 2007 May;44(5):404-9.

12. Azzarito L, Solmon MA, Harrison L Jr. "... If I had a choice, I would..." a feminist poststructuralist perspective on girls in physical education. Res Q Exerc Sport. 2006 Jun;77(2):222-39.

13. Van Daalen C. Girls' experiences in physical education: competition, evaluation, \& degradation. J Sch Nurs. 2005 Apr;21(2):115-21.

14. El Ansari W, Khalil K, Crone D, Stock C. Physical activity and gender differences: correlates of compliance with recommended levels of five forms of physical activity among students at nine universities in Libya. Cent Eur J Public Health. 2014 Jun;22(2):98-105.

15. Cole E, Brown RS. Psychological needs of post-war children in Kosovo: a preliminary analysis. Sch Psychol Int. 2002;23(2):131-47.

16. Ryan RM, Deci EL. Active human nature: self-determination theory and the promotion and maintenance of sport, exercise and health. In: Hagger MS, Chatzisarantis NL, editors. Intrinsic motivation and selfdetermination in exercise and sport. Champaign, Il: Human Kinetics; 2007. p. 1-19.

17. Elder JP, Lytle L, Sallis JF, Young DR, Steckler A, Simons-Morton $\mathrm{D}$, et al. A description of the social-ecological framework used in the trial of activity for adolescent girls (TAAG). Health Educ Res. 2007 Apr;22(2):155-65.

18. Krasniqi G. Parallel system in Kosovo: strengthening ethnic identity through solidarity and common social action. SEEU Rev. 2010;6(1):41-55.

19. Bélanger M, Casey M, Cormier M, Filion AL, Martin G, Aubut S, et al. Maintenance and decline of physical activity during adolescence: insights from a qualitative study. Int J Behav Nutr Phys Act. 2011 Oct 21;8:117. doi: 10.1186/1479-5868-8-117.

20. Torsheim T, Wold B, Samdal O. The teacher and classmate support scale: factor structure, test-retest reliability and validity in samples of 13- and 15-year-old adolescents. Sch Psychol Int. 2000;21(2):195-212.

21. Torsheim T, Samdal O, Rasmussen M, Freeman J, Griebler R, Dür W Cross-national measurement invariance of the teacher and classmate support scale. Soc Indic Res. 2012 Jan;105(1):145-160.

22. Kantanista A, Osiński W, Bronikowski M, Tomczak M. Physical activity of underweight, normal weight and overweight Polish adolescents: the role of classmate and teacher support in physical education. Eur Phy Educ Rev. 2013;19(3):347-59.

23. Cattell RB. The scientific analysis of personality. London: Penguin; 1965.

24. Prochaska JJ, Sallis JF, Long B. A physical activity screening measure for use with adolescents in primary care. Arch Pediatr Adolesc Med. 2001 May;155(5):554-9.

25. Norusis MJ. SPSS 8.0 Guide to data analysis. New York: Prentice Hall; 1998.

26. Spittaels H, Van Cauwenberghe E, Verbestel V, De Meester F, Van Dyck $\mathrm{D}$, Verloigne $\mathrm{M}$, et al. Objectively measured sedentary time and physical activity time across the lifespan: a cross-sectional study in four age groups. Int J Behav Nutr Phys Act. 2012 Dec 18;9:149. doi: 10.1186/1479-58689-149.

27. Clark H. Civil resistance in Kosovo. London: Pluto Press; 2000.

28. Belcher BR, Berrigan D, Dodd KW, Emken BA, Chou CP, Spruijt-Metz D. Physical activity in US youth: effect of race/ethnicity, age, gender, and weight status. Med Sci Sports Exerc. 2010 Dec;42(12):2211-21.

29. Weinberg R, Tenenbaum G, McKenzie A, Jackson S, Anshel M, Grove $\mathrm{R}$, et al. Motivation for youth participation in sport and physical activity: relationships to culture, self-reported activity levels, and gender. Int $\mathrm{J}$ Sport Psychol. 2000;31(3):321-46.

30. Kirby J, Levin KA, Inchley J. Parental and peer influences on physical activity among Scottish adolescents: a longitudinal study. J Phys Act Health. 2011 Aug;8(6):785-93.

31. Brunet J, Sabiston CM, O'Loughlin J, Mathieu ME, Tremblay A, Barnett TA, et al. Perceived parental social support and moderate-to-vigorous physical activity in children at risk of obesity. Res Q Exerc Sport. 2014 Jun;85(2):198-207.

32. Moore LL, Lombardi DA, White MJ, Campbell JL, Oliveria SA, Ellison RC. Influence of parents' physical activity levels on activity levels of young children. J Pediatr. 1991 Feb;118(2):215-9.

33. Eccles JS. The development of children ages 6 to 14. Future Child. 1999 Fall;9(2):30-44

34. Bunke S, Apitzsch E, Bäckström M. The impact of social influence on physical activity among adolescents - a longitudinal study. Eur J Sport Sci. 2013;13(1):86-95.

35. Jurak G, Cooper A, Leskošek B, Kovač M. Long-term effects of 4-year longitudinal school-based physical activity intervention on the physical fitness of children and youth during 7-year follow-up assessment. Cent Eur J Public Health. 2013 Dec;21(4):190-5. 